\title{
Subclass of Harmonic Univalent Functions Associated with the Generalized Mittag-Leffler Type Functions
}

\section{Adnan Ghazy Alamoush}

Faculty of Science, Taibah University, Saudi Arabia

\begin{abstract}
In the present paper, we introduce a new subclass of harmonic functions in the unit disc $U$ defined by using the generalized Mittag-Leffler type functions. Coefficient conditions, extreme points, distortion bounds, convex combination are studied.
\end{abstract}

\section{Introduction}

A continuous complex-valued function $f=u+i v$ defined in a simply complex domain $D$ is said to be harmonic in $D$. In any simply connected domain, we can write $f=h+\bar{g}$, where $h$ and $g$ are analytic in $D$. A necessary and sufficient condition for $f$ to be locally univalent and sense preserving in $D$ is that $\left|h^{\prime}(z)\right|>\left|g^{\prime}(z)\right|, z \in D$.

Clunie and Sheil-Small [7] introduced a class $S H$ of complex valued harmonic maps $f$ which are univalent and sense-preserving in the open unit disk $U=\{z:|z|<1\}$ and assume a normalized representation $f=h+\bar{g}$, where $f(0)=f_{z}(0)-1=0$. Then for $f=h+\bar{g} \in S H$ we may express the analytic functions $h$ and $g$ as

$$
h(z)=z+\sum_{k=2}^{\infty} a_{k} z^{k}, \quad g(z)=\sum_{k=1}^{\infty} b_{k} z^{k}, \quad\left|b_{1}\right|<1 .
$$

Received: October 14, 2019; Accepted: December 12, 2019

2010 Mathematics Subject Classification: 30C45.

Keywords and phrases: analytic functions, univalent function, differential operator, differential subordination, convex functions, structural formula.

Copyright (C) 2020 Adnan Ghazy Alamoush. This is an open access article distributed under the Creative Commons Attribution License, which permits unrestricted use, distribution, and reproduction in any medium, provided the original work is properly cited. 
Later on, Sheil-Small [9] investigated the class $S H$ as well as its geometric subclasses and obtained some coefficient bounds. Since then, there have been several related papers on $\mathrm{SH}$ and its subclasses. Connectivity of geometric functions and hypergeometric functions with harmonic functions is seen through some of these papers ([6], [4], [5], [3], [2], [1]). The Mittag-Leffler and generalized Mittag-Leffler type functions was first introduced by the Swedish mathematician Mittag-Leffler [8] and also studied by Wiman [14]. It is a special function of $z \in C$ which depends on the complex parameter $\alpha$ and is defined by the power series

$$
E_{\alpha}(z)=\sum_{k=0}^{\infty} \frac{z^{k}}{\Gamma(\alpha k+1)}, \alpha \in C, R(\alpha)>0, z \in C .
$$

A first generalization of $E_{\alpha}(z)$ introduced by Wiman [14], is the two-parametric M-L function of $z \in C$, defined by the series

$$
E_{\alpha, \beta}(z)=\sum_{k=0}^{\infty} \frac{z^{k}}{\Gamma(\beta+\alpha k)}, \alpha, \beta \in C, R(\alpha)>0, R(\beta)>0, z \in C .
$$

Prabhakar [10] introduced a three-parametric generalization of $\psi_{\alpha, \beta}^{\gamma}(z)$ defined in (3) as a kernel of certain fractional differential equations in terms of the series

$$
E_{\alpha, \beta}^{\gamma}(z)=\sum_{k=0}^{\infty} \frac{(\gamma)_{k} z^{k}}{k ! \Gamma(\beta+\alpha k)}, \alpha, \beta \in C, R(\alpha)>0, R(\beta)>0, z \in C .
$$

Due to its integral representation $E_{\alpha, \beta}^{\gamma}(z)$ is considered as a special case of Fox's Hfunction as well as of Wright's generalized hypergeometric ${ }_{p} \Psi_{q}$, so called Fox-Wright psi function of $z \in C$. Further extensions of the M-L function to four parameters, Salim [12] introduced the function in the form $\psi_{\alpha, \beta}^{\gamma}(z)$ in the following form

$$
E_{\alpha, \beta}^{\gamma, \delta}(z)=\sum_{k=0}^{\infty} \frac{(\gamma)_{k} z^{k}}{\Gamma(\beta+\alpha k)(\delta)_{k}}
$$

where $\quad \alpha, \beta, \gamma, \delta \in C, \min (R(\alpha), R(\beta)>0, R(\gamma), R(\delta)>0), z \in C$. Recently, Salim and Faraj [13] introduced a new generalization of Mittag-Leffler function associated 
with Weyl fractional integral and differential operators as follow

$$
E_{\alpha, \beta, p}^{\gamma, \delta, q}(z)=\sum_{k=0}^{\infty} \frac{(\gamma)_{q k} z^{k}}{\Gamma(\beta+\alpha k)(\delta)_{p k}},
$$

where $\alpha, \beta, \gamma, \delta \in C, \min (R(\alpha), R(\beta)>0, R(\gamma), R(\delta)>0), z \in C$, with $q, p \in \mathbb{R}_{+}$, $q \leq \mathfrak{R}(\alpha)+p$, and $(\gamma)_{p n}$ denotes an extended variant of the Pochhammer symbol, defined by $(\gamma)_{q n}=\Gamma(\gamma+q n) / \Gamma(\gamma)$.

Corresponding to $E_{\alpha, \beta, p}^{\gamma, \delta, q}(z)$, we define the function $\Theta_{\alpha, \beta, p}^{\gamma, \delta, q}(z)$ by

$$
\begin{aligned}
\Theta_{\alpha, \beta, p}^{\gamma, \delta, q}(z) & =z * E_{\alpha, \beta, p}^{\gamma, \delta, q}(z) \\
& =z+\sum_{k=2}^{\infty} \frac{(\gamma)_{q(k-1)}}{\Gamma[\beta+\alpha(k-1)](\delta)_{p(k-1)}} z^{k} .
\end{aligned}
$$

Now, for $f \in A, m \in \mathbb{N}$, we define the following differential operator: $\Phi_{\gamma, \delta, q, \alpha, \beta, p}^{m} f: A \rightarrow A$ by

$$
\begin{aligned}
& \Psi_{\gamma, \delta, q, \alpha, \beta, p}^{0} f(z)=f(z) * \Theta_{\alpha, \beta, p}^{\gamma, \delta, q}(z), \\
& \Psi_{\gamma, \delta, q, \alpha, \beta, p}^{1} f(z)=z\left[f(z) * \Theta_{\alpha, \beta, p}^{\gamma, \delta, q}(z)\right]^{\prime}, \\
& : \\
& : \\
& (m+1) \Psi_{\gamma, \delta, q, \alpha, \beta, p}^{m+1} f(z)=z\left[\Phi_{\gamma, \delta, q, \alpha, \beta, p}^{m}\right]+m \Psi_{\gamma, \delta, q, \alpha, \beta, p}^{m}, z \in U .
\end{aligned}
$$

Thus it is obvious to see from above that

$$
\Psi_{\gamma, \delta, q, \alpha, \beta, p}^{m} f(z)=z+\sum_{k=2}^{\infty} \frac{(m+1)_{k-1}}{(k-1) !} \frac{(\gamma)_{q(k-1)}}{\Gamma[\beta+\alpha(k-1)](\delta)_{p(k-1)}} a_{k} z^{k}
$$

Note that, when $\alpha=0, \beta=\gamma=\delta=1$, we get Ruscheweyh Operator [11].

Throughout this section, unless otherwise stated, we shall use the notation 


$$
\Phi_{\gamma, \delta, q, \alpha, \beta, p}^{m}=\frac{(m+1)_{k-1}}{(k-1) !} \frac{(\gamma)_{q(k-1)}}{\Gamma[\beta+\alpha(k-1)](\delta)_{p(k-1)}}
$$

Involving the generalized Mittag-Leffler function as defined in (6), for $0 \leq \eta<1, m \in \mathbb{N}, n \in \mathbb{N}_{0}, m>n$ and $z \in U$, let $\operatorname{SH}(m, n, \eta)$ denote the family of harmonic functions $f$ of the form (1.1) such that

$$
\mathfrak{R}\left(\frac{\Psi_{\gamma, \delta, q, \alpha, \beta, p}^{m}}{\Psi_{\gamma, \delta, q, \alpha, \beta, p}^{n}}\right)>\eta
$$

where $\Psi_{\gamma, \delta, q, \alpha, \beta, p}^{m}=\Psi_{\gamma, \delta, q, \alpha, \beta, p}^{m} h(z)+(-1)^{m} \overline{\Psi_{\gamma, \delta, q, \alpha, \beta, p}^{m}} g(z)$.

We let the subclass $\bar{S} H(m, n, \eta)$ consist of harmonic functions $f_{m}=h+\bar{g}_{m}$ in $\bar{S} H(m, n, \eta)$ so that $h$ and $g_{m}$ are of the form

$$
h(z)=z-\sum_{k=2}^{\infty} a_{k} z^{k}, g_{m}(z)=(-1)^{m-1} \sum_{k=1}^{\infty} b_{k} z^{k} .
$$

The class $\bar{S} H(m, n, \eta)$ includes a variety of well-known subclasses of $S H$.

The object of this paper is to examine some generalized Mittag-Leffler function inequalities as a necessary and sufficient condition for univalent harmonic analytic functions associated with certain generalized Mittag-Leffler function to be in the function class $S H(m, n, \eta)$. The coefficient condition for the function class $S H(m, n, \eta)$ is given. Furthermore, we determine extreme points, a distortion theorem, convolution conditions and convex combinations for the functions $f$ in $\bar{S} H(m, n, \eta)$.

\section{Coefficient Bound}

We begin with a sufficient coefficient condition for functions $f$ in $S H(m, n, \eta)$.

Theorem 2.1. Let $f=h+\bar{g}$ be given by (1). If

$$
\sum_{k=1}^{\infty}\left(\frac{\Phi_{\gamma, \delta, q, \alpha, \beta, p}^{m}-\eta \Phi_{\gamma, \delta, q, \alpha, \beta, p}^{m}}{1-\eta}\left|a_{k}\right|\right.
$$




$$
\left.+\frac{\Phi_{\gamma, \delta, q, \alpha, \beta, p}^{m}-(-1)^{m-n} \eta \Phi_{\gamma, \delta, q, \alpha, \beta, p}^{m}}{1-\eta}\left|b_{k}\right|\right) \leq 2
$$

Proof. If $z_{1} \neq z_{2}$, then

$$
\begin{aligned}
\left|\frac{f\left(z_{1}\right)-f\left(z_{2}\right)}{h\left(z_{1}\right)-h\left(z_{2}\right)}\right| \geq 1-\left|\frac{g\left(z_{1}\right)-g\left(z_{2}\right)}{h\left(z_{1}\right)-h\left(z_{2}\right)}\right| \\
=1-\left|\frac{\sum_{k=1}^{\infty} b_{k}\left(z_{1}^{k}-z_{2}^{k}\right)}{\left(z_{1}-z_{2}\right)+\sum_{k=2}^{\infty} a_{k}\left(z_{1}^{k}-z_{2}^{k}\right)}\right| \\
>1-\frac{\sum_{k=1}^{\infty} k\left|b_{k}\right|}{1-\sum_{k=2}^{\infty} k\left|a_{k}\right|} \\
\geq 1-\frac{\sum_{k=1}^{\infty} \Phi_{\gamma, \delta, q, \alpha, \beta, p}^{m}-(-1)^{m-n} \eta \Phi_{\gamma, \delta, q, \alpha, \beta, p}^{n}}{1-\eta} \geq 0, \\
1-\sum_{k=2}^{\infty} \frac{\Phi_{\gamma, \delta, q, \alpha, \beta, p}^{m}-\eta \Phi_{\gamma, \delta, q, \alpha, \beta, p}^{n}\left|a_{k}\right|}{1-\eta}
\end{aligned}
$$

which proves univalence. Note that $f$ is sense-preserving in $U$. This is because

$$
\begin{aligned}
\left|h^{\prime}(z)\right| & =1-\sum_{k=2}^{\infty} k\left|a_{k}\right||z|^{k-1} \\
& >1-\sum_{k=2}^{\infty} \frac{\Phi_{\gamma, \delta, q, \alpha, \beta, p}^{m}-\eta \Phi_{\gamma, \delta, q, \alpha, \beta, p}^{n}}{1-\eta}\left|a_{k}\right| \\
& \geq \sum_{k=1}^{\infty} \frac{\Phi_{\gamma, \delta, q, \alpha, \beta, p}^{m}-(-1)^{m-n} \eta \Phi_{\gamma, \delta, q, \alpha, \beta, p}^{n}}{1-\eta}\left|b_{k}\right| \\
& >\sum_{k=1}^{\infty} \frac{\Phi_{\gamma, \delta, q, \alpha, \beta, p}^{m}-(-1)^{m-n} \eta \Phi_{\gamma, \delta, q, \alpha, \beta, p}^{n}}{1-\eta}\left|b_{k}\right|\left|z^{k}\right| \\
& \geq \sum_{k=1}^{\infty} k\left|b_{k}\right||z|^{k-1} \geq\left|g^{\prime}(z)\right| .
\end{aligned}
$$


Using the fact that $\mathfrak{R}(w)>\eta$ if and only if $|1-\eta+w| \geq|1+\eta-w|$, it suffices to show that

$$
\begin{aligned}
& \left|(1-\eta) \Psi_{\gamma, \delta, q, \alpha, \beta, p}^{n}(z)+\Psi_{\gamma, \delta, q, \alpha, \beta, p}^{m}(z)\right| \\
& \quad-\left|(1+\eta) \Psi_{\gamma, \delta, q, \alpha, \beta, p}^{n}(z)-\Psi_{\gamma, \delta, q, \alpha, \beta, p}^{m}(z)\right|>0 .
\end{aligned}
$$

Substituting the value of $\Phi_{\gamma, \delta, q, \alpha, \beta, p}^{m}(z)$ and $\Phi_{\gamma, \delta, q, \alpha, \beta, p}^{n}(z)$ in (11) yields, by (9), that

$$
\begin{aligned}
& \left|(1-\eta) \Psi_{\gamma, \delta, q, \alpha, \beta, p}^{n}+\Psi_{\gamma, \delta, q, \alpha, \beta, p}^{m}\right|-\left|(1+\eta) \Psi_{\gamma, \delta, q, \alpha, \beta, p}^{n}-\Psi_{\gamma, \delta, q, \alpha, \beta, p}^{m}\right|>0 \\
& =\mid(2-\eta) z+\sum_{k=2}^{\infty}\left[\Phi_{\gamma, \delta, q, \alpha, \beta, p}^{n}(1-\eta)+\Phi_{\gamma, \delta, q, \alpha, \beta, p}^{m}\right] a_{k} z^{k} \\
& +(-1)^{n} \sum_{k=1}^{\infty}\left[\Phi_{\gamma, \delta, q, \alpha, \beta, p}^{n}(1-\eta)+(-1)^{m-n} \Phi_{\gamma, \delta, q, \alpha, \beta, p}^{m}\right] \overline{b_{k} z^{k}} \\
& -\left|-\eta z+\sum_{k=2}^{\infty}\left[\Phi_{\gamma, \delta, q, \alpha, \beta, p}^{m}-(1+\eta) \Phi_{\gamma, \delta, q, \alpha, \beta, p}^{n}\right] a_{k} z^{k}\right| \\
& -(-1)^{n} \sum_{k=1}^{\infty}\left[(-1)^{m-n} \Phi_{\gamma, \delta, q, \alpha, \beta, p}^{m}-(1+\eta) \Phi_{\gamma, \delta, q, \alpha, \beta, p}^{n}\right] \overline{b_{k} z^{k}} \\
& \geq 2(1-\eta)|z|-\left.\sum_{k=2}^{\infty} 2\left[\Phi_{\gamma, \delta, q, \alpha, \beta, p}^{m}-\eta \Phi_{\gamma, \delta, q, \alpha, \beta, p}^{n}\right]\left|a_{k}\right| z\right|^{k} \\
& -\left.\sum_{k=1}^{\infty}\left[(1+\eta) \Phi_{\gamma, \delta, q, \alpha, \beta, p}^{n}+(-1)^{m-n} \eta \Phi_{\gamma, \delta, q, \alpha, \beta, p}^{m}\right]\left|b_{k}\right| z\right|^{k} \\
& -\left.\sum_{k=1}^{\infty}\left|\left[(-1)^{m-n} \Phi_{\gamma, \delta, q, \alpha, \beta, p}^{m}-(1-\eta) \Phi_{\gamma, \delta, q, \alpha, \beta, p}^{n}\right]\right| b_{k}|| z\right|^{k} \\
& =2(1-\eta)|z|\left\{1-\sum_{k=2}^{\infty} \frac{\Phi_{\gamma, \delta, q, \alpha, \beta, p}^{m}-\eta \Phi_{\gamma, \delta, q, \alpha, \beta, p}^{n}}{1-\eta}\left|a_{k}\right||z|^{k-1}\right. \\
& \left.-\sum_{k=1}^{\infty} \frac{\Phi_{\gamma, \delta, q, \alpha, \beta, p}^{m}-(-1)^{m-n} \eta \Phi_{\gamma, \delta, q, \alpha, \beta, p}^{n}}{1-\eta}\left|b_{k}\right||z|^{k-1}\right\} \\
& >2(1-\eta)|z|\left\{1-\left(\sum_{k=2}^{\infty} \frac{\Phi_{\gamma, \delta, q, \alpha, \beta, p}^{m}-\eta \Phi_{\gamma, \delta, q, \alpha, \beta, p}^{n}}{1-\eta}\left|a_{k}\right|\right.\right.
\end{aligned}
$$




$$
\left.\left.+\sum_{k=1}^{\infty} \frac{\Phi_{\gamma, \delta, q, \alpha, \beta, p}^{m}-(-1)^{m-n} \eta \Phi_{\gamma, \delta, q, \alpha, \beta, p}^{n}}{1-\eta}\left|b_{k}\right|\right)\right\}
$$

This last expression is non-negative by (10), and so the proof is complete.

The harmonic function

$$
\begin{aligned}
f(z)= & z+\sum_{k=2}^{\infty} \frac{1-\eta}{\Phi_{\gamma, \delta, q, \alpha, \beta, p}^{m}-\eta \Phi_{\gamma, \delta, q, \alpha, \beta, p}^{n}} x_{k} z^{k} \\
& +\sum_{k=1}^{\infty} \frac{1-\eta}{\Phi_{\gamma, \delta, q, \alpha, \beta, p}^{m}-(-1)^{m-n} \eta \Phi_{\gamma, \delta, q, \alpha, \beta, p}^{n}} \overline{y_{k} z^{k}}
\end{aligned}
$$

where $m \in \mathbb{N}, n \in \mathbb{N}_{0}$ and $\sum_{k=2}^{\infty}\left|x_{k}\right|+\sum_{k=1}^{\infty}\left|y_{k}\right|=1$ shows that the coefficient bound given by (10) is sharp. The functions $f$ of the form (13) is $f \in \operatorname{SH}(m, n, \eta)$, because

$$
\begin{aligned}
& \sum_{k=1}^{\infty}\left(\frac{\Phi_{\gamma, \delta, q, \alpha, \beta, p}^{m}-\eta \Phi_{\gamma, \delta, q, \alpha, \beta, p}^{n}}{1-\eta}\left|a_{k}\right|+\frac{\Phi_{\gamma, \delta, q, \alpha, \beta, p}^{m}-(-1)^{m-n} \eta \Phi_{\gamma, \delta, q, \alpha, \beta, p}^{n}}{1-\eta}\left|b_{k}\right|\right) \\
= & 1+\sum_{k=2}^{\infty}\left|x_{k}\right|+\sum_{k=1}^{\infty}\left|y_{k}\right|=2 .
\end{aligned}
$$

In the following theorem, it is shown that the condition (10) is also necessary for functions $f_{m}=h+\overline{g_{m}}$, where $h$ and $g_{m}$ are of the form (9).

Theorem 2.2. Let $f_{m}=h+\overline{g_{m}}$ be given by (9). Then $f_{m} \in \bar{S} H(m, n, \eta)$ if and only if

$$
\begin{aligned}
& \sum_{k=1}^{\infty}\left[\left(\Phi_{\gamma, \delta, q, \alpha, \beta, p}^{m}-\eta \Phi_{\gamma, \delta, q, \alpha, \beta, p}^{n}\right)\left|a_{k}\right|+\left(\Phi_{\gamma, \delta, q, \alpha, \beta, p}^{m}-(-1)^{m-n} \eta \Phi_{\gamma, \delta, q, \alpha, \beta, p}^{n}\right)\left|b_{k}\right|\right] \\
& \leq 2(1-\eta) .
\end{aligned}
$$

Proof. Since $\bar{S} H(m, n, \eta) \subset \bar{S} H(m, n, \eta)$ we only need to prove the "only if" part of Theorem 2.2. To this end, for functions $f$ of the form (9), we notice that the condition 
(8) is equivalent to

$$
\mathfrak{R}\left\{\begin{array}{c}
(1-\eta) z-\sum_{k=2}^{\infty}\left(\Phi_{\gamma, \delta, q, \alpha, \beta, p}^{m}-\eta \Phi_{\gamma, \delta, q, \alpha, \beta, p}^{n}\right) a_{k} z^{k} \\
+(-1)^{2 m-1} \sum_{k=1}^{\infty}\left(\Phi_{\gamma, \delta, q, \alpha, \beta, p}^{m}-(-1)^{m-n} \eta \Phi_{\gamma, \delta, q, \alpha, \beta, p}^{n}\right) b_{k} \bar{z}^{k} \\
z-\sum_{k=2}^{\infty} \Phi_{\gamma, \delta, q, \alpha, \beta, p}^{n} a_{k} z^{k}+(-1)^{m+n-1} \sum_{k=1}^{\infty} \Phi_{\gamma, \delta, q, \alpha, \beta, p}^{n} b_{k} \bar{z}^{k}
\end{array}\right\} \geq 0 .
$$

The above condition (15) must hold for all values of $z$ on the positive real axes, where, $0 \leq|z|=\mu<1$, we have

$$
\begin{aligned}
1-\eta & -\sum_{k=2}^{\infty}\left(\Phi_{\gamma, \delta, q, \alpha, \beta, p}^{m}-\eta \Phi_{\gamma, \delta, q, \alpha, \beta, p}^{n}\right) a_{k} \mu^{k-1} \\
& -\sum_{k=1}^{\infty}\left(\Phi_{\gamma, \delta, q, \alpha, \beta, p}^{m}-(-1)^{m-n} \eta \Phi_{\gamma, \delta, q, \alpha, \beta, p}^{n}\right) b_{k} \mu^{k-1} \\
-\sum_{k=2}^{\infty} \Phi_{\gamma, \delta, q, \alpha, \beta, p}^{n} a_{k} \mu^{k-1}+(-1)^{m-n} \sum_{k=1}^{\infty} \Phi_{\gamma, \delta, q, \alpha, \beta, p}^{n} b_{k} \mu^{k-1} & \geq 0 .
\end{aligned}
$$

If the condition (14) does not hold, then the numerator in (17) is negative for sufficiently close to 1 . Hence there exists a $z_{0}=\mu_{0}$ in $(0,1)$ for which the quotient in (17) is negative. This contradicts the condition for $f_{m} \in \bar{S} H(m, n, \eta)$ and so the proof is complete.

\section{Distortion Bounds}

In this section, we obtain distortion bounds for functions $f$ in $\bar{S} H(m, n, \eta)$.

Theorem 3.1. Let $f_{m} \in \bar{S} H(m, n, \eta)$. Then for $|z|<1$, we have

$$
\left|f_{m}(z)\right| \leq\left(1+\left|b_{1}\right|\right) \mu+\frac{1}{\left[\Upsilon_{2}\right]^{m}}\left(\frac{(1-\eta)}{\left[\Upsilon_{2}\right]^{m-n}-\eta}-\frac{1-(-1)^{m-n} \eta}{\left[\Upsilon_{2}\right]^{m-n}-\eta}\left|b_{1}\right|\right) \mu^{2}
$$

and

$$
\left|f_{m}(z)\right| \geq\left(1-\left|b_{1}\right|\right) \mu-\frac{1}{\left[\Upsilon_{2}\right]^{m}}\left(\frac{(1-\eta)}{\left[\Upsilon_{2}\right]^{m-n}-\eta}-\frac{1-(-1)^{m-n} \eta}{\left[\Upsilon_{2}\right]^{m-n}-\eta}\left|b_{1}\right|\right) \mu^{2}
$$


where $\left[\Upsilon_{2}\right]^{m}=(m+1)\left(\frac{(\gamma)_{q}}{\Gamma[\beta+\alpha](\delta)_{p}}\right)$.

Proof. We only prove the left-hand inequality. The proof for the right-hand inequality is similar and is thus omitted. Let $f_{m} \in \bar{S} H_{\alpha, \beta, p, n}^{\gamma, \delta, q, m}(\eta)$. Taking the absolute value of $f_{m}$, we have

$$
\begin{aligned}
\left|f_{m}(z)\right|= & \left|z-\sum_{k=2}^{\infty} a_{k} z^{k}+(-1)^{k} \sum_{k=1}^{\infty} b_{k} \bar{z}^{k}\right| \\
\leq & \left(1+\left|b_{1}\right|\right) \mu+\sum_{k=2}^{\infty}\left(\left|a_{k}\right|+\left|b_{k}\right|\right) \mu^{n} \\
\leq & \left(1+\left|b_{1}\right|\right) \mu+\sum_{k=2}^{\infty}\left(\left|a_{k}\right|+\left|b_{k}\right|\right) \mu^{2} \\
= & \left(1+\left|b_{1}\right|\right) \mu+\frac{1-\eta}{\left[\Upsilon_{2}\right]^{m}\left[\Upsilon_{2}\right]^{m-n}-\eta} \\
& \times \sum_{k=2}^{\infty} \frac{\left[\Upsilon_{2}\right]^{m}\left(\left[\Upsilon_{2}\right]^{m-n}-\eta\right)}{1-\eta}\left(\left|a_{k}\right|+\left|b_{k}\right|\right) \mu^{2} \\
\leq & \left(1+\left|b_{1}\right|\right) \mu+\frac{(1-\eta) \mu^{2}}{\left[\Upsilon_{2}\right]^{m}\left(\left[\Upsilon_{2}\right]^{m-n}-\eta\right)} \\
& \times \sum_{k=2}^{\infty}\left(\frac{\Phi_{\gamma, \delta, q, \alpha, \beta, p}^{m}-\eta \Phi_{\gamma, \delta, q, \alpha, \beta, p}^{n}}{1-\eta}\left|a_{k}\right|\right. \\
& \left.+\frac{\Phi_{\gamma, \delta, q, \alpha, \beta, p}^{m}-(-1)^{m-n} \eta \Phi_{\gamma, \delta, q, \alpha, \beta, p}^{n}}{1-\eta}\left|b_{k}\right|\right) \mu^{2} . \\
& \left.+\frac{\Phi_{\gamma, \delta, q, \alpha, \beta, p}^{m}-(-1)^{m-n} \eta \Phi_{\gamma, \delta, q, \alpha, \beta, p}^{n}}{1-\eta}\left|b_{k}\right|\right) \\
\leq & \left(1+\left|b_{1}\right|\right) \mu+\frac{(1-\eta)}{\left[\Upsilon_{2}\right]^{m}\left(\left[\Upsilon_{2}\right]^{m-n}-\eta\right)}\left(\frac{\Phi_{\gamma, \delta, q, \alpha, \beta, p}^{m}-\eta \Phi_{\gamma, \delta, q, \alpha, \beta, p}^{n}}{1-\eta}\left|a_{k}\right|\right. \\
& \\
& \\
& \\
& \\
& \\
&
\end{aligned}
$$

The following covering result follows from the left hand inequality in Theorem 3.1. 


\section{Convolution, Convex Combinations and Extreme Points}

In this section, we show the class $\operatorname{SH}(m, n, \eta)$ is invariant under convolution and convex combination.

For harmonic functions $f$ of the form

$$
f_{m}(z)=z-\sum_{k=2}^{\infty}\left|a_{k}\right| z^{k}+(-1)^{m-1} \sum_{k=1}^{\infty}\left|b_{k}\right| \bar{z}^{k}
$$

and

$$
F_{m}(z)=z-\sum_{k=2}^{\infty}\left|A_{k}\right| z^{k}+(-1)^{m-1} \sum_{k=1}^{\infty}\left|B_{k}\right| \bar{z}^{k}
$$

we define the convolution of $f_{m}(z)$ and $F_{m}(z)$ as

$$
\left(f_{m} * F_{m}\right)(z)=f_{m}(z) * F_{m}(z)=z-\sum_{k=2}^{\infty}\left|a_{k}\right|\left|A_{k}\right| z^{k}+(-1)^{k} \sum_{k=1}^{\infty}\left|b_{k}\right|\left|B_{k}\right| \bar{z}^{k} .
$$

Theorem 4.1. For $0 \leq \rho \leq \eta<1$, let $f_{m} \in \bar{S} H(m, n, \eta)$ and $F_{m} \in \bar{S}(m, n, \rho)$. Then the convolution

$$
f_{m} * F_{m} \in \bar{S} H(m, n, \eta) \subset \bar{S}(m, n, \rho) \text {. }
$$

Proof. Then the convolution $f_{m} * F_{m}$ is given by (17). We wish to show that the coefficients of $f_{m} * F_{m}$ satisfy the required condition given in Theorem 4.1. For $F_{m} \in \bar{S} H(m, n, \rho)$, we note that $\left|A_{k}\right| \leq 1$ and $\left|B_{k}\right| \leq 1$. Now, for the convolution function $f_{m} * F_{m}$, we obtain

$$
\begin{aligned}
& \sum_{k=2}^{\infty} \frac{\Phi_{\gamma, \delta, q, \alpha, \beta, p}^{m}-\rho \Phi_{\gamma, \delta, q, \alpha, \beta, p}^{n}}{1-\rho}\left|a_{k}\right|\left|A_{k}\right| \\
& +\sum_{k=1}^{\infty} \frac{\Phi_{\gamma, \delta, q, \alpha, \beta, p}^{m}-(-1)^{m-n} \rho \Phi_{\gamma, \delta, q, \alpha, \beta, p}^{n}}{1-\rho}\left|b_{k}\right|\left|B_{k}\right| \\
\leq & \sum_{k=2}^{\infty} \frac{\Phi_{\gamma, \delta, q, \alpha, \beta, p}^{m}-\rho \Phi_{\gamma, \delta, q, \alpha, \beta, p}^{n}}{1-\rho}\left|a_{k}\right|
\end{aligned}
$$




$$
\begin{aligned}
& +\sum_{k=1}^{\infty} \frac{\Phi_{\gamma, \delta, q, \alpha, \beta, p}^{m}-(-1)^{m-n} \rho \Phi_{\gamma, \delta, q, \alpha, \beta, p}^{n}}{1-\rho}\left|b_{k}\right| \\
\leq & \sum_{k=2}^{\infty} \frac{\Phi_{\gamma, \delta, q, \alpha, \beta, p}^{m}-\eta \Phi_{\gamma, \delta, q, \alpha, \beta, p}^{n}}{1-\eta}\left|a_{k}\right| \\
& +\sum_{k=1}^{\infty} \frac{\Phi_{\gamma, \delta, q, \alpha, \beta, p}^{m}-(-1)^{m-n} \eta \Phi_{\gamma, \delta, q, \alpha, \beta, p}^{n}}{1-\eta}\left|b_{k}\right|
\end{aligned}
$$

$\leq 1$.

Since $\quad 0 \leq \rho \leq \eta<1, \quad$ and $\quad f_{m} \in \bar{S} H(m, n, \eta), \quad f_{m} * F_{m} \in \bar{S} H(m, n, \eta) \subset$ $\bar{S} H(m, n, \rho)$.

Next, we discuss the convex combinations of the class $\bar{S} H(m, n, \eta)$.

Theorem 4.2. The family $\bar{S} H(m, n, \eta)$ is closed under convex combination.

Proof. For $i=1,2, \ldots$, suppose that $f_{m, j} \in \bar{S} H(m, n, \eta)$, where

$$
f_{m, j}(z)=z-\sum_{k=2}^{\infty}\left|a_{k, j}\right| z^{k}+(-1)^{m-1} \sum_{k=1}^{\infty}\left|b_{k, j}\right| \bar{z}^{k} .
$$

Then by Theorem 2.2,

$$
\begin{aligned}
\sum_{k=1}^{\infty}\left(\frac{\Phi_{\gamma, \delta, q, \alpha, \beta, p}^{m}-\eta \Phi_{\gamma, \delta, q, \alpha, \beta, p}^{n}}{1-\eta} a_{k, j}\right. \\
\left.\quad+\frac{\Phi_{\gamma, \delta, q, \alpha, \beta, p}^{m}-(-1)^{m-n} \eta \Phi_{\gamma, \delta, q, \alpha, \beta, p}^{n}}{1-\eta} b_{k, j}\right) \leq 2 .
\end{aligned}
$$

For $\sum_{j=1}^{\infty} t_{j}=1,0 \leq t_{j}<1$, the convex combination of $f_{m, j}$ may be written as

$$
\begin{aligned}
\sum_{j=1}^{\infty} t_{j} f_{m, j}(z)= & z-\sum_{k=2}^{\infty}\left(\sum_{j=1}^{\infty} t_{j}\left|a_{k, j}\right|\right) z^{k} \\
& +(-1)^{m-1} \sum_{k=1}^{\infty}\left(\sum_{j=1}^{\infty} t_{j}\left|b_{k, j}\right|\right) \bar{z}^{k}
\end{aligned}
$$


Then by (4.3),

$$
\begin{aligned}
& \sum_{k=1}^{\infty}( \frac{\Phi_{\gamma, \delta, q, \alpha, \beta, p}^{m}-\eta \Phi_{\gamma, \delta, q, \alpha, \beta, p}^{n}}{1-\eta} \sum_{j=1}^{\infty} t_{j} a_{k, j} \\
&+\left.\frac{\Phi_{\gamma, \delta, q, \alpha, \beta, p}^{m}-(-1)^{m-n} \eta \Phi_{\gamma, \delta, q, \alpha, \beta, p}^{n}}{1-\eta} \sum_{j=1}^{\infty} t_{j} b_{k, j}\right) \\
&=\sum_{i=1}^{\infty} t_{i}\left\{\sum _ { k = 1 } ^ { \infty } \left(\frac{\Phi_{\gamma, \delta, q, \alpha, \beta, p}^{m}-\eta \Phi_{\gamma, \delta, q, \alpha, \beta, p}^{n}}{1-\eta} a_{k, j}\right.\right. \\
&\left.\left.+\frac{\Phi_{\gamma, \delta, q, \alpha, \beta, p}^{m}-(-1)^{m-n} \eta \Phi_{\gamma, \delta, q, \alpha, \beta, p}^{n}}{1-\eta} b_{k, j}\right)\right\} \\
& \leq 2 \sum_{j=1}^{\infty} t_{j}=2,
\end{aligned}
$$

and therefore

$$
\sum_{j=1}^{\infty} t_{j} f_{m, j}(z) \in \bar{S} H(m, n, \eta)
$$

Corollary 4.3. The class $\bar{S} H(m, n, \eta)$ is closed under convex linear combinations.

Proof. Let the functions $f_{m, j}(z)(j=1,2, \ldots, m)$ defined by (4.2) be in the class $\bar{S} H(m, n, \eta)$. Then the function $\bar{\omega}(z)$ defined by

$$
\varpi(z)=\mu f_{m, j}(z)+(1-\mu) f_{m, j}(z), 0 \leq \mu \leq 1,
$$

is in the class $\bar{S} H(m, n, \eta)$.

Next, we determine the extreme points of closed convex hulls of $\operatorname{SH}(m, n, \eta)$, denoted by $\operatorname{clcoSH}(m, n, \eta)$.

Theorem 4.4. Let $f_{m}$ be given by (10). Then $f_{m} \in \bar{S} H(m, n, \eta)$ if and only if

$$
f_{m}(z)=\sum_{k=1}^{\infty}\left[X_{k} h_{k}(z)+Y_{k} g_{m_{k}}(z)\right],
$$


where

$$
\begin{gathered}
h_{1}(z)=z, h_{k}(z)=z-\frac{1-\eta}{\Phi_{\gamma, \delta, q, \alpha, \beta, p}^{m}-\eta \Phi_{\gamma, \delta, q, \alpha, \beta, p}^{n}} z^{k},(k=2, \ldots), \\
g_{m_{k}}(z)=z+(-1)^{m-1} \frac{1-\eta}{\Phi_{\gamma, \delta, q, \alpha, \beta, p}^{m}-(-1)^{m-n} \eta \Phi_{\gamma, \delta, q, \alpha, \beta, p}^{n}} \bar{z}^{k},(k=1,2, \ldots),
\end{gathered}
$$

$X_{1}=1-\sum_{k=2}^{\infty} X_{k}-\sum_{k=1}^{\infty} Y_{k} \geq 0, X_{k} \geq 0, Y_{k} \geq 0$. In particular, the extreme points of $\operatorname{SH}(m, n, \eta)$ are $\left\{h_{k}\right\}$ and $\left\{g_{m_{k}}\right\}$.

Proof. For functions $f_{m}$ of the form (21) we have

$$
\begin{aligned}
f_{m}(z)= & \sum_{k=1}^{\infty}\left[X_{k} h_{k}(z)+Y_{k} g_{m_{k}}(z)\right] \\
= & \sum_{k=1}^{\infty}\left(X_{k}+Y_{k}\right) z-\sum_{k=2}^{\infty} \frac{1-\eta}{\Phi_{\gamma, \delta, q, \alpha, \beta, p}^{m}-\eta \Phi_{\gamma, \delta, q, \alpha, \beta, p}^{n}} X_{k} z^{k} \\
& +(-1)^{m-1} \sum_{n=1}^{\infty} \frac{1-\eta}{\Phi_{\gamma, \delta, q, \alpha, \beta, p}^{m}-(-1)^{m-n} \eta \Phi_{\gamma, \delta, q, \alpha, \beta, p}^{n}} Y_{k} \bar{z}^{k} .
\end{aligned}
$$

Then

$$
\begin{aligned}
& \sum_{k=2}^{\infty}\left(\frac{\Phi_{\gamma, \delta, q, \alpha, \beta, p}^{m}-\eta \Phi_{\gamma, \delta, q, \alpha, \beta, p}^{n}}{1-\eta}\right) \frac{1-\eta}{\Phi_{\gamma, \delta, q, \alpha, \beta, p}^{m}-\eta \Phi_{\gamma, \delta, q, \alpha, \beta, p}^{n}} X_{k}- \\
& \sum_{k=1}^{\infty}\left(\frac{\Phi_{\gamma, \delta, q, \alpha, \beta, p}^{m}-(-1)^{m-n} \eta \Phi_{\gamma, \delta, q, \alpha, \beta, p}^{n}}{1-\eta}\right) \frac{1-\eta}{\Phi_{\gamma, \delta, q, \alpha, \beta, p}^{m}-(-1)^{m-n} \eta \Phi_{\gamma, \delta, q, \alpha, \beta, p}^{n}} Y_{k} \\
& =\sum_{k=2}^{\infty} X_{n}-\sum_{k=1}^{\infty} Y_{n}=1-X_{1} \leq 1,
\end{aligned}
$$

and so $f_{m} \in \operatorname{clcoSH}(m, n, \eta)$.

Conversely, suppose that $f_{m} \in \operatorname{clcoSH}(m, n, \eta)$. Setting

$$
X_{k}=\frac{\Phi_{\gamma, \delta, q, \alpha, \beta, p}^{m}-\eta \Phi_{\gamma, \delta, q, \alpha, \beta, p}^{n}}{1-\eta}\left|a_{k}\right|, 0 \leq X_{k} \leq 1, k=2, \ldots,
$$




$$
Y_{k}=\frac{\Phi_{\gamma, \delta, q, \alpha, \beta, p}^{m}-(-1)^{m-n} \eta \Phi_{\gamma, \delta, q, \alpha, \beta, p}^{n}}{1-\eta}\left|b_{k}\right|, 0 \leq Y_{k} \leq 1, k=1,2, \ldots
$$

and $X_{1}=1-\sum_{k=2}^{\infty} X_{k}-\sum_{k=1}^{\infty} Y_{k}$, and note that, by Theorem 2.2, $X_{1} \geq 0$. Consequently, we obtain $f_{m}(z)=\sum_{k=1}^{\infty}\left(h_{k}(z) X_{k}+g_{m_{k}}(z) Y_{k}\right)$, as required.

Using Corollary 4.3 we have $\operatorname{clco} S H(m, n, \eta)=\bar{G}_{S} H(m, n, \eta)$. Then the statement of Theorem 4.4 is true for $f \in \bar{G}_{S} H(m, n, \rho)$.

\section{Conclusion}

In this paper, using the Hadamard product or convolution to define a new differential operator involving generalized Mittag-Leffler function. Also, we defined new subclass of univalent functions and established some interesting properties.

\section{References}

[1] O. P. Ahuja, Harmonic starlikeness and convexity of integral operators generated by hypergeometric series, Integral Transforms Spec. Funct. 20(8) (2009), 629-641. https://doi.org/10.1080/10652460902734124

[2] O. P. Ahuja, Planar harmonic convolution operators generated by hypergeometric functions, Integral Transforms Spec. Funct. 18(3) (2007), 165-177. https://doi.org/10.1080/10652460701210227

[3] O. P. Ahuja, Planar harmonic univalent and related mappings, J. Inequal. Pure Appl. Math. 6(4) (2005), 1-18.

[4] A. G. Al Amoush and M. Darus, Harmonic starlike functions with respect to symmetric points, Matematika 32 (2016), 121-131.

[5] A. G. Alamoush and M. Darus, On subclass of harmonic univalent functions associated with convolution of derivative operator, Bull. Calcutta Math. Soc. 106(3) (2014), 153168.

[6] Y. Avc1 and E. Złotkiewicz, On harmonic univalent mappings, Ann. Univ. Mariae CurieSktodowska Sect. A 44 (1990), 1-7.

[7] J. Clunie and T. Sheil-Small, Harmonic univalent functions, Ann. Acad. Sci. Fenn.: Ser. A I Math. 9(3) (1984), 3-25. https://doi.org/10.5186/aasfm.1984.0905 
[8] G. Mittag-Leffler, Sur la Nouvelle function $E_{\alpha}(x)$, Comp. Rend. de l'Acad. des Sci. 137 (1903), 554-558.

[9] T. Sheil-Small, Constants for planar harmonic mappings, J. Lond. Math. Soc. 42(2) (1990), 237-248. https://doi.org/10.1112/jlms/s2-42.2.237

[10] T. R. Prabhakar, A singular integral equation with a generalized Mittag-Leffler function in the kernel, Yokohama Math. J. 19 (1971), 7-15.

[11] S. Ruscheweyh, New criteria for univalent functions, Proc. Amer. Math. Soc. 49 (1975), 109-115. https://doi.org/10.1090/S0002-9939-1975-0367176-1

[12] Tariq O. Salim, Some properties relating to the generalized Mitta-Leffler function, Adv. Appl. Math. Anal. 4 (2009), 21-30.

[13] Tariq O. Salim and Ahmad W. Faraj, A generalization of Mittag-Leffler function and Integral operator associated with fractional calculus, J. Frac. Calc. Appl. 3(5) (2012), 113.

[14] A. Wiman, Über den fundamentalsatz in der Teorie der funktionen $E_{a}(x)$, Acta Math. 29 (1905), 191-201. https://doi.org/10.1007/BF02403202 